\title{
NONSMOOTHABLE, UNSTABLE GROUP ACTIONS
}

\author{
BY
}

DENNIS PIXTON $\left({ }^{1}\right)$

\begin{abstract}
ABSTRACr. For $k>1$ there is a nonempty open set of $C^{1}$ actions of $\mathbf{Z}^{k}$ on $S^{1}$, no element of which is either topologically conjugate to a $C^{2}$ action or structurally stable. The $C^{1}$ closure of this set contains all $C^{2}$ actions which have compact orbits, so no such action is structurally stable in the space of $C^{1}$ actions.
\end{abstract}

1. Introduction. Let $M$ be a smooth manifold, $G$ a Lie group. A $C^{r}$ action of $G$ on $M$ is a homomorphism $\rho: G \rightarrow \operatorname{Diff}^{r}(M)$, the group of $C^{r}$ diffeomorphisms of $M$, such that the evaluation map $(\gamma, p) \rightarrow \rho(\gamma)(p)$ is $C^{r}$. The space of such actions, equipped with the standard $C^{r}$ topology [see \$2], is denoted by $\mathbb{Q}^{r}(G, M)$.

This paper is concerned with the following question: When can a given $C^{r}$ action be approximated by a smoother action? In other words: What is the closure of $\mathbb{Q}^{r+1}(G, M)$ in $\mathbb{Q}^{r}(G, M)$ ? For $G=\mathbf{Z}$ or $\mathbf{R}$ and $r>0$, the classical answer is "everything"-in this case $\mathbb{Q}^{r}(G, M)$ is identified with the space of $C^{r}$ diffeomorphisms or flows.

We restrict ourselves in this paper to the case $G=Z^{k}, k>1$, and $M=S^{1}$. In this case we show that there are many $C^{1}$ actions which cannot be smoothed. Denoting by $\mathbb{P}_{c}^{r}(G, M)$ the subspace of actions which have a compact orbit, we have

Theorem A. Suppose $k>1$. Then $\mathbb{Q}_{c}^{2}\left(\mathbf{Z}^{k}, S^{1}\right)$ is nowhere dense in $\mathbb{Q}^{1}\left(\mathbf{Z}^{k}, S^{1}\right)$. In fact there is an open set $\mathscr{B} \subset \mathbb{Q}^{1}\left(\mathbf{Z}^{k}, S^{1}\right)$ such that

$$
\mathrm{Cl}(\mathscr{B}) \supset \mathbb{Q}_{c}^{2}\left(\mathbf{Z}^{k}, S^{1}\right)
$$

but no action in $\mathscr{B}$ is topologically conjugate to a $C^{2}$ action.

An obvious consequence is that no $C^{2}$ action is structurally stable in $\mathbb{Q}_{c}^{1}\left(\mathbf{Z}^{k}, S^{1}\right)$-this is in contrast to the stability results of [6]. Moreover, we show

THEOREM B. No action in $\mathrm{Cl}(\Re)$ is structurally stable.

It is natural to conjecture that $\mathscr{B}$ is dense in $\mathbb{Q}^{1}\left(Z^{k}, S^{1}\right)$ [and that $\mathbb{Q}_{c}$ may be replaced by $\mathbb{Q}$ in Theorem $A]$. The obstruction to proving this seems to be

Presented to the Society, November 1, 1975; received by the editors December 1, 1975.

AMS (MOS) subject classifications (1970). Primary 58F10; Secondary 57E25, 57D30.

(') Partially supported by NSF Grant MPS74-06731 A01. 
the unsatisfactory state of the closing lemma for group actions. See the comments at the end of $\$ 3$.

As a by-product of the main construction we produce a generalization of the Denjoy diffeomorphism, which is the $k=1$ case of the following.

THEOREM C. For $k \geqslant 1$ there is a free $C^{1}$ action of $Z^{k}$ on $S^{1}$ which is not topologically conjugate to a $C^{2}$ action.

The definitions and proofs for Theorems A and B are in $\$ \$ 2,3$, and the proof of Theorem $\mathrm{C}$ is in $\$ 4$.

Remarks. 1. Rosenberg and Thurston [7] constructed $C^{0}$ actions which cannot be approximated by $C^{2}$ actions, and Hermann [4, p. 412] established a $C^{0}$ version of Theorem $\mathbf{C}$.

2. Our results yield, via suspension, nonsmoothing and instability results for codimension-one foliations, and also for actions of $\mathbf{R}^{k} \times \mathbf{Z}^{l}$ with $k+l>1$.

3. Little seems to be known about the smoothing problem for other groups or manifolds, or even for $C^{r}$ actions of $Z^{k}$ on $S^{1}$ with $r \neq 1$. However, it follows from the existence of stable actions [6] that, for $r>1$, the closure of $\mathbb{Q}^{r+1}\left(\mathbf{Z}^{k}, S^{1}\right)$ in $\mathbb{Q}^{r}\left(\mathbf{Z}^{k}, S^{1}\right)$ has nonempty interior.

2. Essential orbits and bands of orbits. We first recall some definitions. We give $\operatorname{Diff}^{r}(M)$ the uniform $C^{r}$ topology, and $\mathbb{Q}^{r}(G, M)$ the corresponding compact-open topology. If $M$ is compact and $G$ is compactly generated then, for $r<\infty$, it is easy to construct a complete metric for $\mathbb{Q}^{r}(G, M)$. If $G$ is finitely generated, with generators $\gamma_{1}, \ldots, \gamma_{k}$, then a sequence $\rho_{n}$ of actions converges to $\rho_{0}$ in $\mathbb{Q}^{r}(G, M)$ iff the sequences $\rho_{n}\left(\gamma_{i}\right)$ converge to $\rho_{0}\left(\gamma_{i}\right)$ in $\operatorname{Diff}^{r}(M), 1<i<k$. For a fixed action $\rho$ we denote the isotropy subgroup of $p \in M$ by $G_{p}$, the orbit of $A \subset M$ by $O A$, and we usually write $\gamma p$ for $\rho(\gamma)(p), D \gamma(p)$ for the derivative $D[\rho(\gamma)](p)$. Two actions $\rho, \bar{\rho}$ of $G$ on $M$ are topologically conjugate iff there is a homeomorphism $h: M \rightarrow M$ such that $h \rho(\gamma)=\bar{\rho}(\gamma) h$ for all $\gamma \in G$. An action $\rho \in \mathbb{Q}^{r}(G, M)$ is structurally stable iff it is topologically conjugate to all actions in some neighborhood of $\rho$.

For the rest of the paper $G=Z^{k}$ with $k>1$, and, unless otherwise indicated, all actions are $C^{1}$ actions of $G$ on $S^{1}$. The topology on actions is always the $C^{1}$ topology. We think of $S^{1}$ as $\mathbf{R} / \mathbf{Z}$, and use all its standard structure without comment.

We say an orbit $O p$ is essential iff for some compact interval $I$ containing $p$, $\delta_{1}, \ldots, \delta_{k} \in G$, and $s=k-\operatorname{rank} G_{p}$ we have

(a) $\rho\left(\sum_{i=1}^{s} n_{i} \delta_{i}\right) I \cap I \neq \varnothing \Leftrightarrow n_{1}=\cdots=n_{s}=0$,

(b) $\delta_{\mathrm{s}+1}, \ldots, \delta_{k}$ are linearly independent and $\rho\left(\delta_{i}\right) I$ $C$ int $I$ for $s<i \leqslant k$. 
Define $\mathscr{B}_{s}=\left\{\rho \in \mathbb{Q}^{1}\left(G, S^{1}\right):(2.1)\right.$ is satisfied for some $\left.I, \delta_{1}, \ldots, \delta_{k}\right\}$. Since commuting homeomorphisms of a compact interval always have a common fixed point, we see that $\rho \in \mathscr{B}_{s}$ iff $\rho$ has an essential orbit $\theta_{p}$ with rank $G_{p}=k-s$. An orbit $O p$ with rank $G_{p}=k-1$ is called a cylindrical orbit, and we are mainly interested in the set $\mathscr{B}_{1}$ of actions with essential cylindrical orbits. This set is not open; however,

LEMMA 2.1. $\mathscr{B}=\mathscr{B}_{0} \cap \mathscr{B}_{1}$ is open.

Proof. Take $\rho \in \mathscr{B}$. Since $\rho \in \mathscr{B}_{0}$ we have a compact interval $K$ and linearly independent $\gamma_{1}, \ldots, \gamma_{k} \in G$ satisfying

$$
\rho\left(\gamma_{i}\right) K \subset \text { int } K, \quad 1 \leqslant i \leqslant k .
$$

Similarly, since $\rho \in G_{1}$ we have a compact interval $I$ and linearly independent $\delta_{1}, \ldots, \delta_{k} \in G$ satisfying

$$
\begin{array}{ll}
\text { (a) } \rho\left(n \delta_{1}\right) I \cap I=\varnothing & \text { for } n \neq 0, \\
\text { (b) } \rho\left(\delta_{i}\right) I \subset \text { int } I, & 2 \leqslant i \leqslant k .
\end{array}
$$

There is clearly a neighborhood $U$ of $\rho$ such that (2.2) and (2.3b) hold for $\bar{\rho} \in \mathcal{Q}$. Replacing $\delta_{1}$ by $m \delta_{1}$ for some $m \neq 0$ we may assume that $\delta_{1}$ is a linear combination of the $\gamma_{i}$ and that $\rho\left(\delta_{1}\right)$ is orientation preserving. Then, shrinking $Q$ if necessary, and using (2.3a) for $\rho$, we have

$$
\bar{\rho}\left(\delta_{1}\right) I \cap I=\varnothing \text { for } \bar{\rho} \in \mathcal{Q} \text {. }
$$

Take $\bar{\rho} \in \mathcal{Q}$, so $\bar{\rho}\left(\delta_{1}\right)$ is orientation preserving and has a fixed point (since the $\bar{\rho}\left(\gamma_{i}\right)$ do). Thus, if $J$ denotes the component of $S^{1} \backslash$ (fixed point set of $\left.\bar{\rho}\left(\delta_{1}\right)\right)$ which contains $I$, then $\bar{\rho}\left(\delta_{1}\right)$ is a monotone map of the interval $J$ onto itself, so (2.4) implies (2.3a) for $\bar{\rho}$. Therefore $U \subset \mathscr{B}$, so $\mathscr{B}$ is open.

LEMMA 2.2. No action in $\mathfrak{B}_{s}$, for $s \geqslant 1$, is topologically conjugate to a $C^{2}$ action.

Proof. Replacing $\rho$ by $\rho^{2}$, we may assume $\rho$ is orientation preserving. If $\rho$ is topologically conjugate to a $C^{2}$ action then the structure theorem [10] shows: $G_{p}$ is constant on components of $S^{1} \backslash$ (union of compact orbits), and rank $G_{p}<k-2 \Rightarrow \theta p$ is dense in a neighborhood of $p$. If $0<s<k$ and (2.1b) is satisfied then $I$ must meet a compact orbit, contradicting (2.1a). If $s=k$ and (2.1a) is satisfied then any $p \in$ int $I$ has a nowhere dense orbit, contradicting the structure theorem.

Thus the set $\mathscr{B}$ satisfies half of Theorem A-but we have not yet shown that $\mathscr{B}$ is nonempty. To complete the proof of Theorems $A$ and $B$ we require some perturbation theory. First, a definition: Suppose $G_{p}$ is constant for $p$ in some open connected set $V$; then $O V$ is called a band of orbits-a cylindrical band if the orbits are cylindrical. The following three results will be proved in the next section. 
Proposition 2.3 (THICKENING ORBITS). If $\rho \in \mathbb{Q}_{c}^{1}\left(G, S^{1}\right)$ has a cylindrical orbit then it may be approximated by an action with a cylindrical band.

Proposition 2.4 (Cutting Bands). If $\rho \in \mathbb{Q}_{c}^{1}\left(G, S^{1}\right)$ has a cylindrical band $\theta V$, and $p \in V$, then $\rho$ may be approximated by an action $\bar{\rho}$ such that

(a) $\rho$ and $\bar{\rho}$ agree on $S^{1} \backslash \mathcal{O}$,

(b) there is a neighborhood $U$ of $p$ such that only one cylindrical $\bar{\rho}$-orbit meets $U$, and this orbit is essential.

Proposition 2.5. Any $C^{2}$ action in $\mathbb{Q}_{c}^{1}\left(G, S^{1}\right)$ may be approximated by an action in $\mathfrak{B}_{0}$ with a cylindrical band.

Now $\mathrm{Cl}(\mathscr{B}) \supset \mathbb{Q}_{c}^{2}\left(G, S^{1}\right)$ follows immediately from Propositions 2.5 and 2.4, and we have proved Theorem $A$.

Proof OF THEOREM B. It is sufficient to show that no $\rho \in \mathscr{B}$ is structurally stable. If $\rho \in \mathscr{B}$ has no cylindrical bands then we can use Proposition 2.3 to approximate $\rho$ by an action $\bar{\rho}$ with cylindrical bands. Then $\bar{\rho}$ is not conjugate to $\rho$, so $\rho$ is not structurally stable.

Now assume $\rho$ has cylindrical bands. Let $d$ be a complete metric on $\mathbb{Q}^{1}\left(G, S^{1}\right)$ and take $\varepsilon>0$. Set $\rho_{0}=\rho$ and define a sequence $\rho_{j}$ of actions recursively as follows. Let $\theta V$ be a cylindrical band of $\rho_{j}$ such that length of $V$ is maximal, and let $p$ be the midpoint of $V$. Then take $\rho_{j+1}$ satisfying (2.5) with

$$
d\left(\rho_{j+1}, \rho_{j}\right)<\varepsilon / 2^{j+2} .
$$

Set $\bar{\rho}=\lim \rho_{j}$. Then $d(\bar{\rho}, \rho)<\varepsilon$ but $\bar{\rho}$ has no cylindrical bands and so is not conjugate to $\rho$. So, in this case as well, $\rho$ is not structurally stable.

3. Perturbations. We need two preliminary lemmas. The first is a formulation of the well-known procedure of "inserting intervals".

LEMma 3.1. Suppose $Q \subset S^{1}$ is countable, and $\left\{\bar{\lambda}_{q}: q \in Q\right\}$ is a sequence of positive numbers with finite sum $\bar{\lambda}$. Then there is a continuous map $h: S^{1} \rightarrow S^{1}$ such that

(a) $|h(t)-t| \leqslant \bar{\lambda}$ for all $t \in S^{1}$,

(b) For $q \in Q, h^{-1} q=I_{q}$ is an interval of length $\lambda_{q}=$ $\bar{\lambda}_{q}(1+\bar{\lambda})^{-1}$,

(c) $h$ is injective on $S^{1} \backslash h^{-1} Q$, and is affine on the components of $S^{1} \backslash h^{-1} Q$, with derivative $1+\bar{\lambda}$.

The simple construction is essentially contained in [9]. 
Next we introduce a useful group action. Let $\mathbf{R}_{+}$denote the multiplicative group of positive reals.

LEMmA 3.2. There is a $C^{\infty}$ action $\bar{\varphi}$ of $\mathbf{R}_{+}^{3}$ on $D=\left\{(t, \lambda) \in \mathbf{R}^{2}: 0 \leqslant t \leqslant \lambda\right.$, $\lambda>0$ such that

(a) $\bar{\varphi}\left(r, d_{-}, d_{+}\right)(t, \lambda)=\left(\varphi\left(t, \lambda, r, d_{-}, d_{+}\right), \lambda\right)$

for some $\varphi: D \times \mathbf{R}_{+}^{3} \rightarrow \mathbf{R}$,

(b) $\frac{\partial \varphi}{\partial t}\left(t, \lambda, r, d_{-}, d_{+}\right)= \begin{cases}d_{-}, & t=0, \\ d_{+}, & t=\lambda,\end{cases}$

(c) $r^{-1}(\partial \varphi / \partial t)\left(t, \lambda, r, d_{-}, d_{+}\right) \rightarrow 1$ uniformly in $(t, \lambda) \in D$ as $r^{-1} d_{-} \rightarrow 1$ and $r^{-1} d_{+} \rightarrow 1$.

Proof. Let $g:[0,1] \rightarrow \mathbf{R}$ be $C^{\infty}$, with $g^{(n)}(0)=0, n \geqslant 0 ; g(1)=0, g^{\prime}(1)=$ 1. Let $y\left(y_{0}, x\right)$ be the solution of

$$
d y / d x=g(y), \quad y\left(y_{0}, 0\right)=y_{0} .
$$

Then $g(0)=g(1)=0 \Rightarrow y(0, x)=0, y(1, x)=1$ for all $x$. From basic differential equation theory we conclude that $y$ is defined on all of $[0,1] \times \mathbf{R}$, and that $x \rightarrow y(, x)$ is a $C^{\infty}$ action of $\mathbf{R}$ on $[0,1]$.

Next consider $Y=\partial y / \partial y_{0}$; this satisfies

$$
d Y / d x=g^{\prime}\left(y\left(y_{0}, x\right)\right) Y, \quad Y\left(y_{0}, 0\right)=1 .
$$

From $y(1, x)=1$ and $g^{\prime}(1)=1$ we find

$$
\partial y(1, x) / \partial y_{0}=e^{x}
$$

Similarly, from $g^{(n)}(0)=0$ we deduce

$$
\frac{\partial^{n} y}{\partial y_{0}^{n}}(0, x)= \begin{cases}1, & n=1 \\ 0, & n>1 .\end{cases}
$$

Now define, for $s \in[-\lambda / 2, \lambda / 2]$ and $\sigma=\operatorname{sgn}(s)$,

$$
\varphi\left(\frac{\lambda}{2}+s, \lambda, r, d_{-}, d_{+}\right)=\frac{r \lambda}{2}+\sigma y\left(\frac{2 \sigma s}{\lambda}, \log \frac{d_{\sigma}}{r}\right) .
$$

For $s=0$ either choice of $\sigma$ yields $\varphi=r+\lambda / 2$, so $\varphi$ is continuous on $D \times \mathbf{R}_{+}^{3}$. In fact, using (3.4) one readily verifies that $\varphi$ is $C^{\infty}$. Define $\bar{\varphi}$ by (3.2a); it is easy to check that $\bar{\varphi}$ is an action. Compute

$$
\frac{\partial \varphi}{\partial t}=r \frac{\partial y}{\partial y_{0}}\left(\frac{2 \sigma s}{\lambda}, \log \frac{d_{\sigma}}{r}\right) \text {. }
$$

Plugging in $s= \pm \lambda / 2$ and using (3.3) yields (3.2b), and (3.2c) is just the translation of the fact that $\partial y / \partial y_{0}=1$ on $A=[0,1] \times\{0\}$ and is uniformly continuous on a neighborhood of $A$. 
We are now ready to provide the proofs promised in the preceding section. Our main technique in constructing perturbations is, first, to produce a piecewise linear approximation (infinitely many pieces) with suitable uniformity properties, and then to use the action of Lemma 3.2 to smooth off the corners.

Proof of Proposition 2.3. Suppose $\rho \in \mathbb{Q}_{c}^{1}\left(G, S^{1}\right)$ has a cylindrical orbit $O p$. Elementary abelian group theory gives us $\delta \in G$ and a subgroup $H \supset G_{p}$ such that $G=\mathbf{Z} \delta \oplus H$. Since $\rho$ has a compact orbit we can find $m \neq 0$ and $a_{-}, a_{+} \in S^{1}$ such that, for $\sigma= \pm, \theta a_{\sigma}$ is compact and $\rho(n m \delta) p \rightarrow a_{\sigma}$ as $n \rightarrow \sigma \infty$. Set $g=\rho(m \delta), K=\{j \delta+\gamma: 0 \leqslant j<m, \gamma \in H\}$. Any $q \in \theta_{p}$ may be written as $g^{n}(\kappa p), \kappa \in K$.

We want to insert intervals at the points of $\theta p$; for this we need positive numbers $\bar{\lambda}(q)$, depending on $\varepsilon>0$, such that

(a) $(\bar{\lambda}(\gamma q) / \bar{\lambda}(q))|D \gamma(q)|^{-1} \in(1-\varepsilon, 1+\varepsilon)$ for all $q \in \theta p$ and $\gamma$ in some basis of $G$,

(b) the quantity in (a) tends to 1 as $q \rightarrow \theta a_{o}$,

(c) $\bar{\lambda}=\sum\{\bar{\lambda}(q): q \in \theta p\}<\varepsilon$.

To do this, choose $\bar{\lambda}(p)=\bar{\lambda}\left(g^{-1} p\right)>0$ and $N>0$. Define $\bar{\lambda}\left(g^{n} p\right)$ recursively by

$$
\frac{\bar{\lambda}\left(g^{n+\sigma} p\right)}{\bar{\lambda}\left(g^{n} p\right)}= \begin{cases}\left|D g^{\sigma}\left(g^{n} p\right)\right|, & |n| \leqslant N, \\ (n /(n+\sigma))^{2}\left|D g^{\sigma}\left(a_{\sigma}\right)\right|, & |n|>N,\end{cases}
$$

where $\sigma=\operatorname{sgn}(n)$. Then set

$$
\bar{\lambda}\left(g^{n} \kappa p\right)=\left|D \kappa\left(g^{n} p\right)\right| \bar{\lambda}\left(g^{n} p\right), \quad \kappa \in K .
$$

To see that this is well defined it is sufficient to check that $D_{\kappa}(p)=1$ if $\kappa \in G_{p}$. For this, differentiate $g^{n} \kappa=\kappa g^{n}$ and use $\kappa g^{n} p=g^{n} p$ to get $D \kappa(p)=$ $D \kappa\left(g^{n} p\right) \rightarrow D \kappa\left(a_{+}\right)$as $n \rightarrow \infty$. And $D \kappa\left(a_{+}\right)=1$ because $\kappa$ has fixed points in any neighborhood of $a_{+}$. Continuity of $D g^{\sigma}$ and a simple chain rule calculation prove $(3.5 \mathrm{a}, \mathrm{b})$ for $\gamma \in K$ and $N$ sufficiently large. Notice that $\left|D g^{\circ}\left(a_{o}\right)\right|$ $\leq 1$, since $a_{\sigma}$ is a contracting fixed point of $g^{\sigma}$. Then, for fixed $N$ and $\kappa \in K$, $\bar{\lambda}\left(g^{n} \kappa p\right)$ is bounded by (constant) $\cdot n^{-2} \bar{\lambda}(p)$. Since $\{\kappa p: \kappa \in K\}$ is a finite set we see that (3.5c) is satisfied for $\bar{\lambda}(p)$ sufficiently small.

Now get $I_{q}, \lambda_{q}(q \in O p)$ and $h$ from Lemma 3.1. Define $\bar{\rho}(\gamma)$ on $S^{1} \backslash h^{-1} \Theta p$ by the conjugacy $h \bar{\rho}(\gamma)=\rho(\gamma) h$. Recall the map $\varphi$ of Lemma 3.2, and define $\bar{\rho}(\gamma): I_{q} \rightarrow I_{\gamma q}$ by

$$
\varphi\left(, \lambda_{q}, \lambda_{\gamma q} / \lambda_{q},|D \gamma(q)|,|D \gamma(q)|\right)
$$


and appropriate identifications; i.e., use isometries to identify $I_{q}$ with $\left[0, \lambda_{q}\right]$, $I_{\gamma q}$ with $\left[0, \lambda_{\gamma q}\right]$, in such a way that $D[\bar{\rho}(\gamma)]$ has the same sign as $D[\rho(\gamma)]$.

Then $\bar{\rho}$ is an action because of the group properties of $\varphi$. It is continuous and $C^{1}$-except possibly at $h^{-1} \theta a_{ \pm}$-because the definitions fit together properly at the endpoints of the $I_{q}$ [use (3.2b)]. To check that $\bar{\rho}$ is $C^{1}$ on $S^{1}$ we need only check that $D[\bar{\rho}(\gamma)](t)$ has a limit as $t \in \cup I_{q}$ converges to $t_{*} \in$ $h^{-1} 0 a_{ \pm}$, but this is clear from (3.5b) and (3.2c). From (3.5), (3.1), (3.2) we see that $\bar{\rho} \rightarrow \rho$ as $\varepsilon \rightarrow 0$. Finally, notice that, for $\kappa \in G_{p}, \bar{\rho}(\kappa): I_{p} \rightarrow I_{p}$ is given by $\varphi\left(, \lambda_{p}, 1,1,1\right)=$ identity, so the $\bar{\rho}$ orbit of int $I_{p}$ is a cylindrical band.

This completes the proof of Proposition 2.3.

Proof of Proposition 2.4. We have $p \in V$, and $\theta V$ is a cylindrical band. We retain the definitions of $\delta, g, H, K, a_{ \pm}$as in the last proof.

The first step is to approximate $\rho$ by an action which is linear along $O p$. Take $N$ large enough, depending on $\varepsilon>0$, so that, for $t \in g^{n} \kappa V, \kappa, \zeta \in K$, and $\sigma=\operatorname{sgn}(n)$, we have

$$
\left|D \zeta(t) / D \zeta\left(\kappa a_{\sigma}\right)\right| \in(1-\varepsilon, 1+\varepsilon) \text { for }|n| \geqslant N \text {, and } \rightarrow 1 \text { as }|n| \rightarrow \infty .
$$

Set $K_{N}=\{n m \delta+\kappa:|n|<N, \kappa \in K\}$ and select an interval $I_{p} \subset V$, with midpoint $p$, such that

$$
|D \zeta(t) / D \zeta(\gamma p)| \in(1-\varepsilon, 1+\varepsilon) \text { for } t \in \gamma I_{p}, \gamma, \zeta \in K_{N} .
$$

This is possible by uniform continuity of the finitely many functions $D \zeta \mid \gamma V$ $\left(\zeta, \gamma \in K_{N}\right)$. For $q=\gamma p \in O p$ set $I_{q}=\gamma I_{p}, \lambda_{q}=$ length of $I_{q}$. Let $b_{p}^{ \pm}$be the endpoints of $I_{p}$ and set $b_{q}^{ \pm}=\gamma b_{p}^{ \pm}$; let $I_{q}^{0}$ be the middle third of the interval $I_{q}$. Now define $\bar{\rho}(\gamma)=\rho(\gamma)$ off of $\theta I_{p}$; define $\bar{\rho}(\gamma)$ to be affine from $I_{q}^{0}$ onto $I_{\gamma q}^{0}$, and define $\rho(\gamma)$ on the other thirds of $I_{q}$ by $\varphi\left(, \lambda_{q} / 3, \lambda_{\gamma q} / \lambda_{q},\left|D \gamma\left(b_{q}^{ \pm}\right)\right|\right.$, $\left.\lambda_{\gamma q} / \lambda_{q}\right)$ with appropriate identifications. Do this so the definitions match up (including derivatives) at the boundaries. Using (3.7), (3.8) and the properties of $\varphi$, we see that $\bar{\rho}$ is a $C^{1}$ action and $\bar{\rho} \rightarrow \rho$ as $\varepsilon \rightarrow 0$.

So replace $\rho$ by $\bar{\rho}$; then

$$
|D \gamma(t)|=\lambda_{\gamma q} / \lambda_{q} \text { for } t \in I_{q}^{0}, \gamma \in G .
$$

We shall require positive numbers $\mu_{n j}(n, j \in \mathbf{Z})$, depending on $\varepsilon>0$, with the following properties:

(a) $\sum_{j \in \mathbf{Z}} \mu_{n j}=1$, all $n$,

(b) $\left|\mu_{n+1, j} / \mu_{n j}-1\right|,\left|\mu_{n j+1} / \mu_{n j}-1\right|<\varepsilon$,

(c) the quantities in (b) approach 0 as $|n|+|j| \rightarrow \infty$.

For this, let $n>0$ and set 


$$
\bar{\mu}_{n j}= \begin{cases}\frac{3}{5} \frac{n+j^{2}}{n(n+1)(n+2)}, & |j| \leqslant n, \\ \bar{\mu}_{|j|, n}, & |j| \geqslant n .\end{cases}
$$

Then $(3.10 \mathrm{a}, \mathrm{c})$ are easy to check for $\bar{\mu}_{n j}$-standard summation formulas work for (a), and straightforward estimates for (c). Define $\mu_{n j}=\bar{\mu}_{N+|n|} j$. For $N$ sufficiently large, (b) is also satisfied.

Now let $J_{p}$ be one half of the interval $I_{p}^{0}$ [so that $p$ is an endpoint of $J_{p}$ ] and set $J_{\gamma p}=\gamma J_{p}$, so $J_{q}$ has length $\lambda_{q} / 6$. Recall the decomposition $G=\mathbf{Z} \delta \oplus H$; let $\pi: G \rightarrow \mathbf{Z}$ be the projection on the first factor. Partition $J_{\gamma p}$ into subintervals $J_{\gamma p, j}(j \in \mathbf{Z})$ with $\lambda_{\gamma p, j}=\mu_{\pi \gamma, j} \cdot \lambda_{\gamma p} / 6$. Do this so that $J_{\gamma p, j}$ is between $J_{\gamma p, j-1}$ and $J_{\gamma p, j+1}$ and

$$
J_{\gamma p, j} \rightarrow \gamma p \text { as } j \rightarrow+\infty .
$$

Set $\delta_{1}=\delta$, let $\delta_{2}, \ldots, \delta_{k}$ be a basis for $H$, and define a homomorphism $\tau$ : $G \rightarrow \mathbf{Z}$ by $\delta_{i} \rightarrow 1$, all $i$. Then define $\bar{\rho}(\gamma)=\rho(\gamma)$ off of $\theta J_{p}$, and define $\bar{\rho}(\gamma)$ : $J_{q j} \rightarrow J_{\gamma q j+r \gamma}$ by means of

$$
\varphi\left(, \lambda_{q j}, r_{j}, r_{j}, r_{j+1}\right) \text { with } r_{i}=\lambda_{\gamma q, i+\gamma \gamma} / \lambda_{q, i} .
$$

It is, as usual, easy to check that $\rho$ is a continuous action which is $C^{1}$ on $S^{1} \backslash \partial \Theta J_{p}$. Since $\partial \Theta J_{p}$ is countable, $\bar{\rho}$ is a $C^{1}$ action iff

$$
D[\bar{\rho}(\gamma)](t) \rightarrow D[\rho(\gamma)]\left(t_{*}\right)
$$

as $t \in \mathcal{O} J_{p}$ converges to $t_{*} \in \partial \Theta J_{p}$. But, with $t \in J_{\zeta p}$, we have

$$
r_{i}=\frac{\lambda_{(\zeta+\gamma) p, i+\tau \gamma}}{\lambda_{\zeta p, i}}=\frac{\lambda_{(\zeta+\gamma) p}}{\lambda_{\zeta p}} \frac{\mu_{\pi(\zeta+\pi \gamma, i+\tau \gamma)}}{\mu_{\pi \zeta, i}} .
$$

Then, for fixed $\gamma$, as $t \rightarrow t_{*}$ the first factor approaches $\left|D[\rho(\gamma)]\left(t_{*}\right)\right|$-see (3.9)-and $|\pi \zeta|+|i| \rightarrow \infty$, so the second factor tends to 1 by a finite number of applications of (3.10c). Thus, using (3.2c), we have (3.12). Similar arguments, applied with $\gamma=\delta_{1}, \ldots, \delta_{k}$ and using (3.10b), show that $\bar{\rho} \rightarrow \rho$ as $\varepsilon \rightarrow 0$.

Now from (3.11) it is clear that no cylindrical orbits pass through int $J_{p}$; in fact, one may readily check that $\theta$ (int $J_{p}$ ) is a band of orbits with common isotropy group $=G_{p} \cap \operatorname{ker} \tau$, and so of rank $k-2$.

Finally, perform the same construction on $\theta J_{p}^{\prime}$ where $J_{p}^{\prime}$ is the other half of $I_{p}^{0}$, so that (3.11) holds for $J_{\gamma p}^{\prime}$. Then, for the perturbed action, no cylindrical orbit except $O p$ meets int $I_{p}^{0}$, and from (3.11) it follows that $O p$ is essential.

This completes the proof of Proposition 2.4.

Proof of Proposition 2.5. Suppose $\rho$ has a flat compact orbit $O p$, i.e., $|D \gamma(p)|=1$ for all $\gamma \in G_{p}$. First, imitate the proof of Proposition 2.3 to obtain a band of compact orbits. Then imitate the proof of Proposition 2.4 to introduce a cylindrical band and an essential compact orbit: 
The alternative is that all compact orbits are hyperbolic, i.e., whenever $\theta p$ is compact there is some $\gamma \in G_{p}$ with $|D \gamma(p)| \neq 1$. In this case the argument is based on Kopell [3]. Let $I_{1}, \ldots, I_{r}$ be the closures of the components of $S^{1} \backslash$ (union of the compact orbits), and set $H=\left\{\gamma \in G: \gamma I_{1}=I_{1}\right\}$. If $\gamma \in H$ reversed orientation then $\gamma$ would have a unique fixed point in int $I_{1}$, whose orbit would be compact. Thus all $\gamma \in H$ preserve orientation, whence we deduce $\gamma I_{j}=I_{j}, 1 \leqslant j \leqslant r$. Using [3, Corollary 1] we can find $\delta \in H$ such that $|D \delta(p)| \neq 1$ for all $p$ with compact orbits. Set

$$
e_{j}=\left\{h \in \operatorname{Diff}^{1}\left(I_{j}\right): h \rho(\delta)=\rho(\delta) h \text { and } D h>0\right\} .
$$

By [3, Lemma 3] there is a closed subgroup $\Gamma_{j} \subset \mathbf{R}$ and a unique injective action $\psi_{j} \in \mathbb{Q}^{1}\left(\Gamma_{j}, I_{j}\right)$ such that $\psi_{j}(1)=\rho(\delta) \mid I_{j}$ and $\mathcal{C}_{j}=\psi_{j}\left(\Gamma_{j}\right)$. Gluing together the $\psi_{j}^{\prime}$ 's gives a $C^{1}$ action $\psi$ of $\Gamma=\Gamma_{1} \cap \cdots \cap \Gamma_{r}$ on $S^{1}$. Note that if $f: I_{i} \rightarrow I_{j}$ is a diffeomorphism commuting with $\rho(\delta)$ then $\mathcal{C}_{i}=f^{-1} \mathcal{C}_{j} f$; it follows, by uniqueness, that $\psi_{i}(t)=f^{-1} \psi_{j}(t) f$. So for all $\gamma \in G$ and $t \in \Gamma$ we have $\rho(\gamma) \psi(t)=\psi(t) \rho(\gamma)$.

Define a homomorphism $\alpha: H \rightarrow \Gamma$ by $\rho(\gamma)=\psi(\alpha(\gamma))$. If $\alpha(H)$ is cyclic then it is easy to see that, for $p \in \operatorname{int} I_{j}, H_{p}=\operatorname{ker} \alpha$, so rank $H_{p}=k-1$. And clearly $G_{p}=H_{p}$, so $\Theta$ (int $I_{j}$ ) is a cylindrical band. If $\alpha(H)$ is not cyclic then $\Gamma=\mathbf{R}$. Then $\alpha$ extends to $\alpha_{1}: G \rightarrow \mathbf{R}$, which may be approximated (compact-open topology) by $\bar{\alpha}_{1}: G \rightarrow \mathbf{R}$ such that $\bar{\alpha}_{1}(G)$ is cyclic. Define

$$
\bar{\rho}(\gamma)=\rho(\gamma) \psi\left[\bar{\alpha}_{1}(\gamma)-\alpha_{1}(\gamma)\right] .
$$

Then $\bar{\rho}$ is a $C^{1}$ action and $\bar{\rho}(\gamma)=\psi\left(\bar{\alpha}_{1}(\gamma)\right)$ for $\gamma \in H$. So, by the argument above, $\bar{\rho}$ has cylindrical bands. Also, $\bar{\rho} \rightarrow \rho$ as $\bar{\alpha}_{1} \rightarrow \alpha_{1}$, so we are done with Proposition 2.5.

REMARK. We are left with the open question: Is $\mathscr{B}$ dense in $\mathcal{Q}^{1}\left(G, S^{1}\right)$ ? This resolves itself into two problems: (a) whether $\mathscr{B}$ is dense in $\mathbb{Q}_{c}^{1}\left(G, S^{1}\right)$, and (b) whether $\mathbb{Q}_{c}^{1}\left(G, S^{1}\right)$ is dense in $\mathbb{Q}^{1}\left(G, S^{1}\right)$. Problem (b) is essentially a weak closing lemma, which is well known for $G=\mathbf{R}$ or $\mathbf{Z}$ [5], and false for general groups and manifolds [2]; there remains some meager encouragement when $G$ is abelian [8], but even for $\mathbf{Z}^{2}$ acting on $S^{1}$ the answer is unknown. Problem (a) asks whether any action with a compact orbit can be approximated by an action with a cylindrical orbit, and so is closely related to the closing lemma. The proof of Proposition 2.5 relies heavily on Kopell's work on centralizers, which definitely fails (by the examples in this paper) for $C^{1}$ diffeomorphisms.

4. Denjoy actions. We now present the proof of Theorem C, which is quite similar to the arguments in \$3. Recall that an action is free iff all isotropy groups are trivial.

We start with a free linear action $\rho$; in particular, all orbits are dense and 


$$
D \gamma(t)=1, \text { all } t \in S^{1} \text { and } \gamma \in G \text {. }
$$

In terms of some basis $\delta_{1}, \ldots, \delta_{k}$ for $G$ we write $\left|\Sigma n_{i} \delta_{i}\right|=\Sigma\left|n_{i}\right|$. Select positive numbers $\bar{\lambda}_{\gamma}$, for $\gamma \in G$, such that

$$
\text { (a) } \sum_{\gamma \in G} \bar{\lambda}_{\gamma}<\infty \text {. }
$$

(b) For any $\delta \in G, \bar{\lambda}_{\gamma+\delta} / \bar{\lambda}_{\gamma} \rightarrow 1$ as $|\gamma| \rightarrow \infty$.

For example, $\bar{\lambda}_{\gamma}=(1+|\gamma|)^{-k-1}$ works. Choose $p \in S^{1}$ and apply Lemma 3.1 to $\theta_{p}$ to obtain $h, \lambda_{\gamma}, I_{\gamma}$. Now define $\bar{\rho}(\delta)$ on $S^{1} \backslash h^{-1} \theta_{p}$ by $h \bar{\rho}(\delta)=$ $\rho(\delta) h$, and define $\bar{\rho}(\delta): I_{\gamma} \rightarrow I_{\gamma+\delta}$, using the action of Lemma 3.2 and appropriate identifications, by $\varphi\left(, \lambda_{\gamma}, \lambda_{\gamma+\delta} / \lambda_{\gamma}, 1,1\right)$. As in the last section, it is easy to see that $\bar{\rho}$ is a continuous action, $C^{1}$ on $U=U$ (int $I_{\gamma}$ ). To see that it is $C^{1}$ on $S^{1}$ it is sufficient to check (a) $D[\bar{\rho}(\delta)](t)=1$ for $t \in \partial U$, and (b) $D[\bar{\rho}(\delta)](t) \rightarrow 1$ as $t \in U$ converges to $t_{*} \in \partial U$. But (a) follows from (4.1), (4.2a) exactly as in Denjoy's original construction [1], [9]. And note that $t \in I_{\gamma}$ and $t \rightarrow t_{*} \in \partial U \Rightarrow|\gamma| \rightarrow \infty$, so (b) follows from (4.2b) and (3.2c).

It is clear that $\bar{\rho}$ is still a free action. But every $t \in U$ has an essential orbit [note that (2.1b) is vacuous] so $\bar{\rho}$ is not conjugate to any $C^{2}$ action, by Lemma 2.2 , and we are done.

\section{REFERENCES}

1. A. Denjoy, Sur les courbes définies par les équations différentielles à la surface du tore, J. Math. Pures Appl. (9) 11 (1932), 333-375.

2. M. W. Hirsch, Foliations and noncompact transformation groups, Bull. Amer. Math. Soc. 76 (1970), 1020-1023. MR 45 \#1189.

3. N. Kopell, Commuting diffeomorphisms, Proc. Sympos. Pure Math., vol. 14, Amer. Math. Soc., Providence, R. I., 1970, pp. 165-184. MR 42 \# 5285.

4. H. B. Lawson, Jr., Foliations, Bull. Amer. Math. Soc. 80 (1974), 369-418. MR 49 \#8031.

5. C. C. Pugh, The closing lemma, Amer. J. Math. 89 (1967), 956-1009, MR 37 \#2256.

6. H. Rosenberg and R. Roussarie, Some remarks on stability of foliations, J. Differential Geometry 10 (1975), 207-219.

7. H. Rosenberg and W. Thurston, Some remarks on foliations, Dynamical Systems (Proc. Sympos, Univ. of Bahia, Salvador, 1971), Academic Press, New York, 1973, pp. 463-478. MR 49 \#3974.

8. R. Roussarie and D. Weil, Extension du 'closing lemma' aux actions de $R^{2}$ sur les variétés de $\operatorname{dim}=3$, J. Differential Equations 8 (1970), 202-228. MR 41 \#9289.

9. P. A. Schweitzer, Counterexamples to the Seifert conjecture and opening closed leaves of foliations (appendix), Ann. of Math. (2) 100 (1974), 386-400. MR 50 \#8557.

10. J. W. Wood, Foliated $S^{1}$-bundles and diffeomorphisms of $S^{1}$, Dynamical Systems (Proc. Sympos., Univ. of Bahia, Salvador, 1971), Academic Press, New York, 1973, pp. 671-681. MR 49 \#8036.

Department of Mathematics, Northwestern University, Evanston, Illinois 60201

Current address: Department of Mathematics, Indiana University, Bloomington, Indiana 47401 